\title{
Fortschritte in der Behandlung des
}

Diabetes mellitus.

Von Dr. J. Strauss in Frankfurt a. M.

I. Diätetische Therapie.

1. Kohlehydrate.

In den letzten Jahren sind - teils auf dem Boden empirisch gewonnener Tatsachen, teils auf Grund theoretischer Voraussetzungen 
- mehrfach Vorschläge für die Behandlung des Diabetes mellitus gemacht worden, die das Gemeinsame haben, daß bei der speziell empfohlenen Diätform eine größere Menge von Kohlehydraten zugeführt wird. Inwieweit unsere Anschauungen über die diätetische Behandlıng einzelner Formen des Diabetes durch die Einführung der verschiedenen "Kohlehydratkuren" eine Abänderung erfahren haben, soll an der Hand der einzelnen Vorschläge besprochen werden.

\section{a) Milchkuren.}

Nach den Mitteilungen von Winternitz und Strasser (17) macht eine strenge Milchkur in kürzester Zeit, meist schon nach 48 Stunden die Mehrzahl der Diabetiker zuckerfrei oder vermindert zum mindesten die Zuckeransscheidung sehr beträchtlich. Dies gelte sowohl für die bei jugendlichen Individuen vorkommenden, meist für sehr schwer gehaltenen Diabetesformen als alch für die bei sehr fettleibigen Personen oder nach Trauma oder Chok auftretenden Zuckerkrankheiten. Auch in Fällen von Diabetes. die bei strenger Diabetesdiät nicht zuckerfrei wurden, soll unter strenger Milchkur eine weitere Abnahme, selbst vollständiges Verschwinden der Zuckerausscheidung zur Beobachtung kommen. Wenn beim Uebergang von der Milchkur zu gemischter Kost Zucker wieder anftrete, so bringe ihn die Einleitung einer neuerlichen strengen Milchkur wieder zum vollständigen Verschwinden. Einzelne Diabetiker werden durch die Milchkur vollständig geheilt und bleiben es bei gemischter Kost mit reichlicher Zufuhr von Amylaceen. Kolisch (39), der auf Grund noch zu erwähnender theoretischer Erwägnngen für die Behandlung des Diabetes eine quantitative Reduktion der Nahrung anstrebt, sodaß bei niedrigstem Kalorienwert Stoffwechselgleichgewicht garantiert wird, und der namentlich bei den schweren Formen des Diabetes derjenigen Kost den Vorzug gibt, welche bei derselben Wirkung auf die Glycosurie mehr Kohlehydrate zuführt, redet - neben der strengen vegetarianischen Kost - auch der Milchdiät das Wort.

Die Empfehlung der Milchkuren bei Diabetes ist weder neu, noch blieb sie unwidersprochen. Naunyn (71) berichtet, daß Rollo über 1 Liter Milch täglich bei Diabetes gab und daß vor drei Jahrzehnten D onkin die ausschließliche Ernährung mit Milch als unfehlbares Heilmittel des Diabetes pries. Daß die reine Milchdiät gelegentlich bei Diabetikern in Betracht kommt, hat auch A. Hoffmann in v. Leydens Handbuch der Ernährungstherapie (33) erwähnt. Ihrer Anwendung stehen aber gewichtige Bedenken entgegen. Na un y bemerkt zwar bezüglich der Toleranz der Diabetiker gegenüber der Milchzufuhr, daß geringere Mengen, bis zı 1/2 Liter, oft selbst in recht schweren Fällen genommen werden können, ohne daß die Glycosurie ansteigt, daß aber im übrigen die Diabetischen sich dem Milchzucker gegenüber verschieden verhalten; in praxi sei deshalb die Wirkıng der Milch auf die Glycosurie stets im Einzelfall erst festzustellen; zu beachten sei dabei, daß sich ihr ungünstiger Einfluß erst nach längere Zeit fortgesetztem Genu $\beta$ bemerkbar zu machen brauche. Nach den Untersuchungen von H. Strauss (101) läuft übrigens die variable Toleranz der Diabetiker für Milchzucker auf die Frage hinaus, wie sich ihre assimilierenden Organe gegenüber dem Traubenzucker verhalten. Die Diabetiker vertragen den ungespaltenen Milchzucker zwar gut, scheiden ihn aber nach der Spaltung leicht aus, und die Zuckerausscheidung nach Milchzuckerzufuhr gestaltet sich in den einzelnen Fällen verschieden, je nach der Intensität des auch im Darminhalt der Erwachsenen von H. Strauss nachgewiesenen Milchzucker spaltenden Ferments. Die Angaben von Winternitz und Strasser über den Einfluß reiner Milchdiät auf diabetische Glycosurien wurden in der Nothnagelschen Klinik auf Veranlassung von W. Schlesinger durch A. B e rge r (4) einer speziellen Nachprïfung unterzogen: Ein Fall von leichtem Diabetes, in dem bei $50 \mathrm{~g}$ Brot und $1 / 4$ Liter Milch kein Zucker ausgeschieden wurde, zeigte bei 3 Liter Milch eine Glycosurie von $3 \mathrm{~g}$. In einem zweiten, nach seinem ganzen klinischen Verhalten gleichfalls der leichten Form zuzurechnenden Falle, in dem bei reichlicher Kohlehydratzufuhr (4 Semmeln, 2 Liter Milch) $64 \mathrm{~g}$ Zucker ausgeschieden wurden, sank die Glycosurie bei reiner Milchdiät (2 bis 3 Liter) auf wenige Gramm. In einem dritten Falle von leichtem Diabetes verringerte sich die Zuckerausscheidung bei der offenbar ungenügenden Ernährung mit 2 Liter Milch bloß um ein Geringes. Drei Diabetiker mit schwerer Form der Glycosurie reagierten auf die Milchdiät mit sehr starker Zuckerausscheidung. Ein bereits zuckerfrei gewesener Patient schied sofort $84 \mathrm{~g}$ Zucker ans, und - entsprechend dem von Naunyn hervorgehobenen Verhalten - überdauerte in diesem Falle die Zuckerausscheidung die Darreichung der Milch noch um zwei Tage. F. Hirschfeld (31) berichtet davon, daß er in einzelnen Fällen von leichtem Diabetes unter dem Einfluß von Milchkuren bisweilen ein Verschwinden des Zuckers konstatiert habe, doch sei die Verringerung der Glycosurie keine so große gewesen, daß er daraufhin eine besondere Heilkraft der Milch annehmen konnte. F. Blumenthal (8) ist bei der Durchführung von Milchkuren bei Diabetikern aufgefallen, daß in manchen Fällen die Zuckerausscheidung danach stieg, daß andererseits allerdings in einem Falle die Acetonurie schwand. Le nn é(46) fand für das Gros der Diabetiker die Milch als Diätform unzuträglich, er zieht sie - in der von Winternitz empfohlenen Form - als
Entziehungsdiät, aber nicht als Diabetikerdiät in Frage. v. Noorden, der die Angaben von Winternitz über die günstige Einwirkung ausschließlicher Milchdiät auf die Glycosurie in einzelnen Fällen bestätigen konnte (76) (s. hieriiber unter Haferkuren), erklärt es für einen bedauerlichen theoretischen Irrtum, wenn systematische Milchkuren - in praxi sind diejenigen, welche auf die Empfehlung von Winternitz und Strasser die Milchkuren aufgenommen haben, offenbar über das von den Autoren selbst gesteckte Ziel hinausgegangen - als Idealnahrung für Diabetiker hingestellt werden (73). In Uebereinstimmung mit $\mathrm{N}$ a un y netont v. Noorden, daß die Frage der Regelung der Milchzufuhr bei Diabetikern gar nicht allgemein zu beantworten sei, sondern für jeden einzelnen Fall besonders geprïft werden müsse. Unter allen Umständen mïsse bei der Behandlung der leichten Formen des Diabetes vermieden werden, daß unter der Milchzufnhr Glycosurie auftrete. Keinesfalls befürwortet v. Noorden, Milch als einziges Nahrungsmittel an Diabetiker generell zil verabreichen. selbst dann nicht, wenn trotz 4 Liter Milch pro die - der für erwachsene Menschen zwecks Erhaltung des stofflichen Bestandes notwendigen Mindestmenge - die Glycosurie wegbleibe, da ausschließliche Milchkuren sehr häufig, speziell anch bei Diabetikern, eine sehr schwer zu bekämpfende Appetitlosigkeit hinterlassen. Im ïbrigen rät v. Noorden, in den Fullen von leichtem Diabetes. in denen dadurch keine Glykosurie ausgelöst wird. ausgiebigen Gebrauch von der Milch in der Weise zu machen, daß bei der Durchführung einer speziellen Milchkur ein wesentlicher Teil des Nahrungsbedürfnisses durch Milch (resp. Kefir, saure Milch, Diabetikermilch, Kumys, Rahm) gedeckt wird.

Eine neuerdings von seiten Strassers (100) zum Kapitel der Milchkuren gelegentlich geäußerte Bemerkung unterscheidet sich iibrigens von der urspriinglichen Empfehlıng der Milchkuren durch Winternitz und Strasser insofern, als Strasser in Uebereinstimmung mit einer zweiten Mitteilıng (99) angibt. daß es ,gegen die Milchkuren refraktäre Diabetiker wohl auch sehr viele gibt, und daß oft die Unterernährung. als welche sich die Milchkur (mit Zufuhr von $2 \frac{1}{2}-3$ Liter Milch pro die) darstellt, nicht gitt vertragen wird".

b) Kartoffelknren.

Der Empfehlung der Kartoffelkuren durch Mossé $(69,70)$ liegt folgendes Tatsachenmaterial zugrunde: Mossé ersetzte in Fällen von Diabetes das Brot während einer mehrwöchigen Periode durch Kartoffeln. Die Kranken erhielten $1500 \mathrm{~g}$ Kartoffeln, die unter Rücksichtnahme auf ihre wechselnde Zusammensetzung den Bestandteilen von zirka $500 \mathrm{~g}$ Weißbrot gleich gesetzt wurden. In anderen untersuchten Fällen betrug die Kartoffelmenge zwischen $1 / 2$ und $3 \mathrm{~kg}$, meistens $1-1 \frac{1}{2} \mathrm{~kg}$, immer das dieifache Gewicht des vergleichsweise gegebenen Brotquantıms. Dabei ergab die unter den nötigen Kautelen ausgeführte Beobachtıng, daß während der Kartoffeldiät, während der andere Kohlehydrate als Kartoffeln nicht gereicht wurden, eine Verringerung der Glycosurie eintrat, mit der ein Nachlassen des Durstes, eine Abnahme der Urinmenge und eine Hebung des Allgemeinbefindens einherging. Die Falle, die Mossé untersuchte, gehörten größtenteils der „arthritischen“ Form der Krankheit an, zum Teil dem „nervösen“ Diabetes, zum Teil aber auch dem "mageren" (pancreatischen) Diabetes. In einem Teil der Fälle zeigte sich auch ein günstigerer Verlauf von Wunden, resp. chirurgischen Komplikationen. Der Grund für den giinstigen Einfluß der Kartoffeldiät auf Zuckerausscheidung und Befinden der Diabetiker ist nach Mossé nicht etwa in einer mangelhaften Resorption oder Umbildung der in den Kartoffeln enthaltenen Kohlehydrate zu suchen, da in zwei Fällen während abwechselnder Darreichung von Brot und äquivalenter Kartoffelmengen die in den Faeces enthaltenen Kohlehydrate im wesentlichen als gleich befunden wurden. Mos sé führt vielmehr den Vorzıg, den die Kartoffel in der Ernährung des Diabetikers vor dem Brot hat, auf den großen Gehalt der Kartoffel an Wasser und Kalisalzen zurück. Durch die vermehrte Wasserzufuhr werde dem quälenden Durstgefühl abgeholfen, die Besserung der anderen Symptome des Diabetes sei dagegen als eine Folge der Salzwirkung anzusehen. Von 23 Fällen, die zum Teil unter abwechselnder Zufuhr von Brot und Kartoffeln beobachtet wurden, wurde nur bei einem ein Vorteil der Kartoffeldiät vermißt. Den Mosséschen Erfolgen ähnliche berichtet in einer kurzen Diskussionsbemerkung Offer (79), der einer Anzahl von Diabetikern Kartoffeln bis zu $500 \mathrm{~g}$ zur Standartkost zulegte. In diesen Fällen war die Zuckerausscheidung geringer, als man nach der Kohlehydratzufuhr erwarten sollte.

c) Haferkuren.

v. Noorden (76) machte auf der Naturforscherversammlung in Karlsbad Mitteilıng von der bemerkenswerten Tatsache. daß in vielen, besonders in schweren Fällen von Diabetes der Hafer mindestens doppelt so gut vertragen wird wie äquivalente Mengen von Brot. Allgemein traf dieses Verhalten jedoch nicht $\mathrm{zu}$, in einigen Fällen wurde bei der Haferzufuhr mehr Zucker ausgeschieden als bei Darreichung von Brot mit gleichem Stärkegehalt. In einer Reihe von Fällen stellte sich nun das paradoxe Verhalten heraus, daß unter allmählich steigen- 
der Haferzufuhr die Glycosurie nicht nur nicht anstieg, sondern unter gleichzeitiger Abnahme der Acetonurie und des Ammoniakgehalts des Urins - sich verminderte. Die günstigen, die Toleranz steigernden Resultate der Haferfütterung wurden aber nur da erzielt, wo der Hafer in der Kost bei weitem vorhelrschte und nur dann, wenn neben dem Hafer keine anderen Kohlehydrate gegeben wurden. Worauf diese paradoxe Wirkung der Haferzufuhr auf die Glycosurie Diabetischer beruht, wollte v. Noorden nicht mit Bestimmtheit entscheiden, hielt es aber - ähnlich der von Mossé für die Wirkung der Kartoffeldiät gegebenen Erklärung - nicht für unmöglich, daß die Beigabe anderer im Hafer enthaltener Stoffe, etwa der Salze, dem steigernden EinfluB der Kohlehydrate auf die Glycosurie entgegenarbeite. Gleich Mossé weist auch v. Noorden, gestützt auf den Ausfall der Kotanalysen, die Möglichkeit einer schlechteren Resorption zurück. W. Schlesinger (89) hat unlängst zur Erklärung des Erfolges der Kohlehydratkuren (Hafer, Kartoffeln, Milch) die Ver mutung ausgesprochen, daß unter der Einfuhr großer Mengen eines isoliert in den Darm eingebrachten Kohlehydratträgers möglicherweise im Darm die Entwicklung bestimmter, spezifischer Gärungserreger im Uebermaß erfolgt, so daß eine weitgehende Zerstörung des Kohlehydrats schon vor seiner Resorption erfolgt. Daß diese Vermutung zutrifft, ist deshalb nicht wahrscheinlich, weil dann schwer zu verstehen wäre, daß die Haferkur bei den verschiedenen Formen des Diabetes, wie wir sehen werden, verschieden vertragen wird. Man könnte bezüglich der Erklärung des Erfolges der Haferkur noch an eine weitere Möglichkeit denken, an die nämlich, daß die Aufbesserung der Toleranz für Kohlehydrate durch Hafer eine Folge der durch die Kohlehydratzufuhr bedingten Verringerung der Acetonämie sein könnte. Natürlich ist das nur eine Hypothese, die zur Voraussetzung hätte, daß im klinischen Versuch — oder im Tierexperiment beim entpancreasten Hund - die Einverleibung von Aceton eine Schädigung des Kohlehydratstoffwechsels zur Folge hat. Untersuchungen über diese Frage liegen, soweit ich sehe, nicht vor, doch fand ich einige für diese Frage verwertbare Versuchsprotokolle, die Untersuchungen über das Schicksal des dem diabetischen Organismus zugeführten Acetons, respektive von Oxybuttersäure entstammen und in denen die Höhe der an den Versuchstagen ausgeschiedenen Zuckermenge vermerkt ist. Teils ist hiernach die Glycosurie nach Darreichung von Aceton, respektive von Oxybuttersäure in der Tat etwas höher [zwei Fälle von Diabetes (Leo Schwartz [93])], teils ist das um. gekehrte Verhalten festzustellen (ein Fall von Diabetes [H. StrauB und H. Philippsohn]) (102), in dem an zwei von drei Versuchstagen unter Aceton geringere Zuckermengen ausgeschieden wurden), teils ist eine Einwirkung nicht ersichtlich (ein Versuch Minkowskis [62] mit der Darreichung von oxybuttersaurem Natron beim entpancreasten Hund). Immerhin kann das vorliegende Material nicht als ausreichend für die Entscheidung der aufgeworfenen Frage angesehen werden, besonders, da es über das Verhalten der diabetischen Glycosurie unter längere Zeit fortgesetzter Einverleibung von Aceton - derartige, mit kleineren Mengen von Aceton (4-6 g) anzustellende klinische Untersuchungen sind a priori nicht unzulässig, da das Aceton ja früher zu therapeutischen $\mathrm{Zwecken}$ gegeben wurde ( $\mathrm{KuBmaul}$ ) - nicht Auskunft gibt. Muß demnach die Frage, ob die diabetische Glycosurie und die Acetonämie in einem gewissen Abhängigkeitsverhältnis zueinander stehen, offen bleiben, so wäre andererseits mit ihrer Bejahung bezüglich der Einwirkung der Haferkur nicht erklärt, weshalb der Hafer besser vertragen wird als Brot und daß die besondere Wirkung nur bei Zufuhr einer einzigen Kohlehydratart zustande kommt.

Was die Durchführbarkeit der Haferernährung anlangt, so berichtet v. Noorden, daß die Behandlung mit Rücksicht darauf, daß bei den Patienten sich relativ rasch Widerwillen gegen die einseitige Ernährungsform herausstellte, meistens nicht länger als 8-14 Tage fortgesetzt werden konnte. Bei der Rückkehr zu der gewöhnlichen Diabetesdiät machte v. Noorden dann häufig die unangenehme Erfahrung, daß die Toleranz für andere Arten von Nahrungsmitteln, z. B Brot, nicht wesentlich gestiegen war und die Glycosurie sich rasch wieder vermehrte. Noch ungünstiger war der Verlauf in einzelnen Fällen, in denen im AnschluB an die Haferkur trotz Verabreichung mittlerer Mengen von Kohlehydraten die Acetonmengen in jäher Weise anstiegen, so daß man mit der Möglichkeit eines Comas, das allerdings noch abzuwenden war, rechnen mußte.

In einer neueren Mitteilung (77), die sich auf die Beobachtung von etwa 100 Fällen von Diabetes unter Haferzufuhr stützt gruppiert v. Noorden die Erfolge der Haferkur folgendermaßen: 1. Durch die Haferkur wurden in Fällen, in denen es unter Anwendung strenger Diät nicht gelungen war, den Harn zuckerfrei zu machen, überraschende Erfolge erzielt, die bei Innehaltung des üblichen Diätschemas nicht so rasch oder nicht in derselben Weise zu erzielen gewesen wären. Es war in diesen Fällen schließlich möglich, neben der gewöhnlichen strengen Diabetesdiät ansehnliche Mengen verschiedener Kohlehydrate zu geben, ohne daB es wieder zu Glycosurie kam. 2. Es trat zwar eine günstige Reaktion auf die Haferkur ein, aber eine nachhaltige und wesentliche Steigerang der Toleranz wurde nicht erzielt. Immerhin wurde durch rechtzeitige Einleitung der Haferkur in einem genauer geschilderten Fall dieser Art das drohende Coma abgewendet. Bei den Fällen beider Gruppen fiel auf, daß unter der Haferzufuhr eime erhebliche Eiweißmast erzielt wurde. 3. Die Toleranz für Kohlehydrate während der Haferkur unterschied sich nicht wesentlich von der in der vorangehenden und nachfolgenden Zeit bestehenden. Dagegen sank die Acetonurie und behielt den niedrigen Wert bei, als wieder die frühere Diät befolgt wurde. 4. In einer nicht geringen Zahl von Fällen wurde die Glycosurie nicht vermindert, sondern vermehrt oder nahm doch den Verlauf, den man nach den bisherigen Anschauungen über Kohlehydratzufuhr in schweren Fällen erwartet hätte. 5. Leichte Fälle von Diabetes ohne Diaceturie vertrugen die Haferkur sehr schlecht und büßten in der Regel an Toleranz ein.

Für die Technik derHaferkur, die inzwischen auch auf derEwaldschen Klinik durch Sigel (95) mit gutem Erfolg angewendet wurde, gibt v. Noorden folgende Vorschriften: Knorrsches Hafermehl oder Hohenlohesche Haferflocken werden sehr lange mit Wasser und etwas Salz gekocht; während des Kochens wird Butter und ein vegetabilisches Eiweiß oder nach Abkühlung geschlagenes Eiereiweiß hinzugesetzt (gewöhnlich wurde Roborat oder ein aus der Bremer Brotfabrik bezogenes Reiseiweiß, das in bezug auf den Geschmack den Vorzug zu verdienen schien, genommen). Die gewöhnlichen Tagesmengen, sofort von Beginn der Haferkur an, betragen $250 \mathrm{~g}$ Hafer, $100 \mathrm{~g}$ Eiweiß, $300 \mathrm{~g}$ Butter. Die mit diesen Hilfsmitteln bereitete Suppe wird zweistündlich verabreicht; daneben ist etwas Kognak oder Wein und etwas starker, schwarzer Kaffee gestattet.

Die genauere Charakteristik der Fälle mit gutem Erfolg der Haferkuren läßt sich nach dem Urteile v. Noordens zwar vorläufig noch nicht geben, doch sind die Fälle von denjenigen Diabetesfällen zu trennen, in denen auch die Gewährung einer größeren Menge verschiedenartiger Kohlehydrate einen besseren Erfolg nach sich zog als eine strenge Diät, da die günstige, weitergehende und auch andere Formen des Diabetes umfassende Wirkung der Haferkuren sich an die Verabreichung eines einzigen Kohlehydrates gebunden zeigte. Analog der Haferzufuhr wirkt nach neueren Mitteilungen v. Noordens (78) ungeschälte gestampfte Gerste, ferner ließ sich zeigen, daß Brot aus ganzem Korn, sogenanntes Schrotbrot (z. B. das D.-K.-Brot von Rademann), viel besser vertragen wird als Tafelbrot mit entspechendem Amylumgehalt. In der Regel schnitt jedoch Hafer am besten ab, insbesondere hatten die Haferkuren vor den Kartoffelkuren Mossés und den seinerzeit durch v. Düring empfohlenen Reiskuren, wie die vergleichende Prüfung ergab, den Vorteil einer stärkeren Einwirkung voraus. $\mathrm{Ob}$ die paradoxe Einwirkung einzelner Stärkesorten durch Verschiedenheiten in der chemischen Konstitution bedingt ist oder ob in den Rohvegetabilien Stoffe enthalten sind, welche die Glycosurie vermindern, läßt v. Noorden auch neuerdings unentschieden.

Für die Praxis beachtenswert und für die Indikationsstellung zu Haferkuren von Bedeutung dürfte sein, daß es doch durchweg schwerere Fälle von Diabetes - mit Diaceturie - zu sein scheinen, in denen mit $\mathrm{Hafer}$ günstige Erfolge erzielt wurden, und daB die leichten Fälle-ohne Diaceturie - entsprechend ihrem sonstigen Verhalten gegen Kohlehydratzufuhr auch unter $\mathrm{Haf}$ a eine Verschlechterung ihrer Toleranz erfuhren. Man wird also keineswegs schematisch jegliche Form des Diabetes der Haferkur unterziehen dürfen. Ausdrücklich warnt v. Noorden hievor. „Denn neben wenigen Fällen, denen man damit wirklich nützt, stehen viele, für die die Behandlung ohne besonderen Vorteil ist und eine noch größere Zahl, denen man damit ernstlich schaden würde." Hält man sich in praxi für die Anwendung der Haferkur ausschließlich an schwerere Fälle, so wird man unter Berücktichtigung des vorliegenden Tatsachenmaterials verlangen müssen, daß man sich außerdem in jedem einzelnen Falle durch tägliche Kontrolle der Zucker-und Acetonausscheidung vergewissert, inwieweit in dem betreffenden Falle durch die Haferkur Nutzen gestiftet wird, oder ob man Veranlassung hat, mit der Haferkur abzubrechen und zu anderen Maßnahmen uberzugehen. Es ist begreiflich, daß derartige ambulante Untersuchungen nur ausnahmsweise bei solchen Patienten durchgeführt werden könnten, deren Gewissenhaftigkeit vorher erprobt ist, daß im allgemeinen hiefür klinische Behandlung als unbedingt erforderlich erachtet werden muß. v. Noorden bedient sich der Haferkur auf Grund seiner inzwischen gesammelten weiteren Beobachtungen vorzugsweise bei schwersten Glycosurien mit starker Acetonurie, insbesondere in Fällen, in denen bei Beschränkung, beziehungsweise bei Entziehung der Kohlehydrate die Summe der Ácetonkörper nicht wieder sinkt oder gar fortwährend ansteigt. „Man würde sich der Gefahr des Coma diabeticum nähern, wenn man trotzdem bei jenen strengen Vorschriften beharren wollte. Wenn auch die Ausschaltung der Kohlehydrate beim Diabetiker nicht die einzige Ursache der Acidosis ist (cf. L. Mohr [64]), so wissen wir doch jetzt, daß sie eine mächtige und eine der wesentlichsten Ursachen derselben darstellt. Jedenfalls ist Tatsache, daß man durch freigebige Gewährung von Kohlehydraten diese wieder rückgängig machen kann. “ v. Noorden hebt für die Behandlung gerade dieser Fälle die Ueberlegenheit des Hafers gegenüber der Zufuhr anderer Kohlehydrate hervor und L. M ohr 
bezeichnet die Haferkur als das beste Mittel, das wir gegen schwere Grade der Acidosis besitzen. Auch für die Diätbehandlung des Coma diabeticum gibt $\mathbf{v}$. Noorden der Hafersuppe gegenüber der früher (73) von ihm empfohlenen ausschließlichen Milchzufuhr den Vorzug, da diese schneller die A cetonkörper des Harns vermindert. „Immerhin hat man die Wahl zwischen den beiden", nur rät v. Noorden, das eine oder das andere, nicht beide nebeneinander zu reichen. Bezüglich der Milchzufuhr bei starker Acidosis, respektive beim Coma nimmt Naunyn (71) einen ähnlichen Standpunkt ein, wie v. Noorden ihn früher vertreten hat, indem er rät, dann, wenn die Acetessigsäurereaktion stärker wird und so weit geht, daß der Urin bei Zusatz von Eisenchlorid schwarz wird, vorsichtigerweise Milch zuzulegen (unter Verminderung der Fleischnahrung), und weiter fortfährt: „Sobald irgend welche Zeichen von Coma auftreten, muß man fast stets alle Diätbeschränkungen aufgeben, und wieder ist es die Milch, mit der man anch unter diesen Umständen am besten fährt.“

Zur Illustrierung der Wirkung der Haferkur mag der folgende, von Herrn Prof. v. Noorden mir zwecks Mitteilung freundlichst überlassene, in dessen Privatklinik neuerdings zur Beobachtung gekommene Fall dienen.

\begin{tabular}{|c|c|c|c|c|c|c|c|}
\hline 些 & Kost & 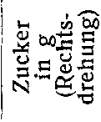 & 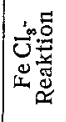 & 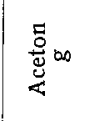 & $z \infty \infty$ & ${\frac{T^{\infty}}{Z}}^{\infty}$ & 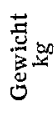 \\
\hline $\begin{array}{l}11 \\
12 \\
13 \\
14 \\
15 \\
16 \\
17 \\
18 \\
19 \\
20 \\
21 \\
22 \\
23 \\
24 \\
25\end{array}$ & 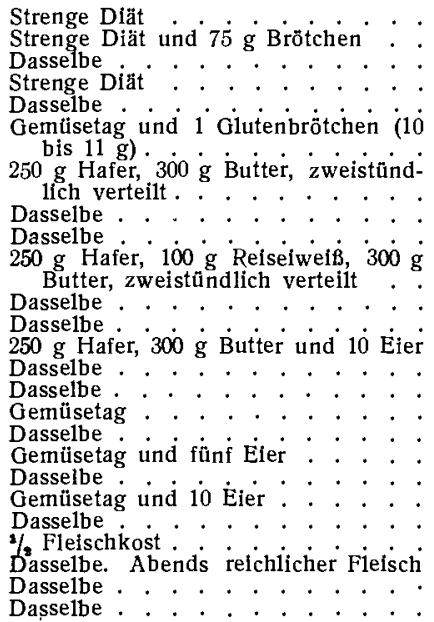 & $\begin{array}{c}15,75 \\
22,05 \\
19,32 \\
11,20 \\
7,23 \\
3,9 \\
11,64 \\
17,8 \\
7,2 \\
0,8 \\
\text { Spuren } \\
0,85 \\
2,88 \\
1,40 \\
4,375 \\
4,84 \\
8,40 \\
6,54 \\
16,60\end{array}$ & $\begin{array}{l}0 \\
0 \\
0 \\
0 \\
+ \\
+ \\
+ \\
+ \\
+ \\
+ \\
+ \\
+ \\
+ \\
+ \\
+\end{array}$ & $\begin{array}{l}0,386 \\
0,540 \\
0,650 \\
1,390 \\
0,902 \\
\\
0,240 \\
0,168 \\
0,110 \\
0,128 \\
\text { spuren } \\
0,156 \\
0,407 \\
0,480 \\
0,806 \\
0,968 \\
1,098 \\
1,497 \\
1,229 \\
1,236 \\
0,770 \\
1,260 \\
1,344 \\
1,92 \\
1,677\end{array}$ & $\begin{array}{r}5,44 \\
5,32 \\
- \\
9,66 \\
12,09 \\
13,52 \\
8,94 \\
13,18 \\
11,18 \\
12,57 \\
10,28 \\
11,68 \\
22,89 \\
16,13 \\
15,46\end{array}$ & $\begin{array}{l}1,312 \\
0,718 \\
0,799 \\
1,723 \\
1,296 \\
1,006 \\
0,574 \\
0,988 \\
2,070 \\
2,112 \\
2,060 \\
1,663 \\
1,577 \\
2,94 \\
2,112 \\
2,489\end{array}$ & $\begin{array}{l}85,3 \\
\overline{-} \\
\overline{85,4} \\
\overline{-} \\
\overline{84,6} \\
\overline{-} \\
\overline{83,4} \\
\overline{-} \\
\overline{84}, 5\end{array}$ \\
\hline
\end{tabular}

In dem mitgeteilten Falle sank die Glycosurie, die bei strenger Diät auf $32 \mathrm{~g}$ heruntergegangen war, nach Einleitung der Haferkur bis auf zirka $4 \mathrm{~g}$, und verschwand bei nachfolgender Einschaltung von zwei Gemüsetagen bis auf Spuren. Die Acetonurie sank von 0,38 bis $1,39 \mathrm{~g}$ bis auf Spuren, stieg jedoch allerdings bei weiterer Fortsetzung der Haferkur unter gleichzeitigem Auftreten einer positiven Eisenchloridreaktion wieder an. Bemerkenswert an dem Falle ist, daß die Glycosurie unter Hafer ihr tiefstes Niveau erreichte, als neben $250 \mathrm{~g}$ Hafer und $300 \mathrm{~g}$ Butter $100 \mathrm{~g}$ Reiseiweiß, deren Gehalt an Eiweiß nach Mitteilung der Fabrik durchschnittlich zirka $90 \mathrm{~g}$ beträgt, gereicht wurden und sich bei der nachfolgenden Zufuhr eines geringeren Eiweißquantums (10 Eier = zirka $56 \mathrm{~g}$ Eiweiß) sogar etwas höher gestaltete. Man wird daher in dem vorliegenden Falle ebenso wie in den früheren Fällen v. Noordens, in denen neben Hafer und Butter $100 \mathrm{~g}$ Roborat oder Reiseiweiß gegeben wurde, die giinstige Wirkung des Hafers nicht ohne weiteres auf eine gleichzeitige geringere Eiwejßzufuhr zuruckführen können.

Líteratur. 1. E. Allard, Zeitschrift für klinische Medizinn Bd. 45. - 2. Ba um, Blätter für kllnische Hydrotherapie 1902 , No. 3. - 3. A. B erg e r, Inauguraldissertation,
Halle. Ref. in Virchow-Hirschs Jahresberichten 1898, Bd. 2, S. 39. - 4. A. Berger, Wiener Halle. Ref. in Virchow-Hirschs Jahresberichten 1898, Bd. 2, S. 39. - 4. A. Berger, Wiener
klinische Rundschau 1900, No. 31. - 5. Alexander Biado brzews ki , Gazeta lekarska

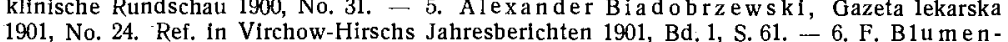

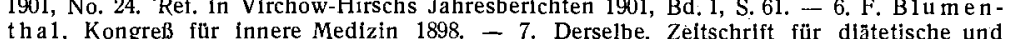
physikalische Theraple 1899. - 8. Derselbe, Zeitschrift für diătetische und physikalische physikalische Theraple 1899. - 8. Derselbe, Zeitschrift für diătetische und physikalische Therapie 1901. - 9. Derselbe, Deutsche medizinische Wochenschrift 1903, No. $51 .-$
10. M. Boígey, Archiv. gén. de méd., Jan. 1903. Ref. Im Zentrablblatt für Stoffwechsel10. M. Boigg y, Archiv. gén. de méd., Jan. 1903. Ref. Im Zentralblatt für Stoffwechsel-
und Verdauungskrankheiten 1903, No. 7. - 11. H. B rü n i ng, Inauguraldissertation, Halle. Ref. in Virchow-Hirschs Jahresberichten 1898, Bd. 2, S. 39. - 12. Ca n tan I, zitiert nach v. Merıng. - 13. Cassa ët, La Semaine médicale 1895, S. 376. - 14. Cohnhel m, Zeitschrift für physiologische Chemle, September 1903. - 15. C. Croftan, American journal of medical science 1902. Ref. im Zentralblatt für Stoffwechsel- und Verdauungskrankheitten 1902, S. 227. - 16. W. Croner, Deutsche medizinische Wochenschrift 1903, No. 45. 17. Elch h orst, Therapeutische Monatshefte 1902. - 18. Hilt on Fagge, Guys hospital records 1874, Bd. 19. Zitiert nach $M$ ag nus-Levy, Archiv für experimentelle Pathologie und Pharmakologie 1901. - 19. Falt a, Korrespondenzblatt für Schweizer Aerzte 1903, No. 22 - 20. Derselbe, Kongreß für innere Medizin 1904 - 21. F a us t, Klinisch-therapeutische Wochenschrift 1898 Zitiert nach Welntraud im Goldscheider Jakobschen (aï Handbuch. - 22. Oeel huy de n, Zelschnth Wel physlologische Chennie Bd. 23 und 24. Zitiert nach Leo Schward. 23. Olibert et Carnsot, Semaine médicale 1896, S. 489 Gaz. hebd. de méd. et de chir. 1902, No. 81. Ref. im Zentralblatt für Stoffivectisel- und

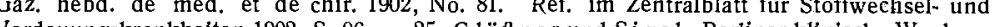
Verdauungskrankheiten 1902, S. 96. - 25. G 1 äß n er und Sig e1, Berliner klinische Wochenschrift 1904, No. 17. - 26. Goli ine r, Therapeutische Monatshefte 1903, No. 8. - 27. Grube, Münchener medizinische Wochenschrift 1895, No. 21. - 28. Derselbe, Therapeutische Monatshefte 1896, S. 259. - 29. Derselbe, Zeitschrifit für diătettsche und physikalische Therapie, Mai 1902. - - 30. Ra hel H irsch, Hofmeisters Beitrạge, Oktober 1903, und Disser- tation, Strafburg, September 1903. - 31. F. Hirsch feld, Die Zuckerkrankheit, Leipzig 1902. - 32. Hans Hirsch feld, Fortschritte der Medizin 1901, Na. 1. - 33. A. Hoff-
mann, Leydens Handbuch der Ernăhrungstheraple, 1. Auflage 1899, Bd. 1, S. 583. (Fortsetzung folgt.) 